\title{
Parámetros productivos del cultivo de cachama blanca Piaractus orinoquensis, en jaulas flotantes
}

\section{Productive parameters of white cachama culture, Piaractus orinoquensis, in floating cages}

\author{
Jorge Andrés Cuan-Barrera'; Sandra Liliana Parada-Guevara ${ }^{2}$; Ricardo Murillo-Pacheco ${ }^{3}$; Juan Antonio Ramírez-Merlano ${ }^{4 *}$
}

\begin{abstract}
${ }^{1}$ Médico Veterinario y Zootecnista. Universidad de los Llanos, Grupo de Investigación sobre reproducción y toxicología de organismos acuáticos (GRITOX), Instituto de Acuicultura y Pesca de la Universidad de los Llanos (IALL). Villavicencio - Meta, Colombia; e-mail: jorge.cuan@unillanos.edu.co; iD https://orcid.org/0000-00031896-8370

${ }^{2}$ Bióloga; M.Sc. Corporación Kotsala, Grupo de Investigación en Educación, Biodiversidad y Sistemas Productivos. Universidad de los Llanos. Villavicencio - Meta, Colombia; e-mail: salipa11@gmail.com; (iD) https://orcid.org/0000-0002-5266-8112

${ }^{3}$ Médico Veterinario y Zootecnista, M.Sc. Corporación Kotsala, Grupo de Investigación en Educación, Biodiversidad y Sistemas Productivos. Universidad de los Llanos. Villavicencio - Meta, Colombia; e-mail: rimupa@yahoo.com; iD https://orcid.org/0000-0002-9785-4699

${ }^{4}$ Profesional en Acuicultura, M.Sc., Ph.D. Universidad de los Llanos, Grupo de Investigación sobre reproducción y toxicología de organismos acuáticos (GRITOX), Instituto de Acuicultura y Pesca de la Universidad de los Llanos (IALL). Villavicencio - Meta, Colombia; e-mail: jramrezmerlano@unillanos.edu.co; iD https://orcid. org/0000-0001-9712-6678

*autor para correspondencia: jramrezmerlano@unillanos.edu.co
\end{abstract}

Cómo citar: Cuan-Barrera, J.A.; Parada-Guevara, S.L.; Murillo-Pacheco, R.; Ramírez-Merlano, J.A. 2021. Parámetros productivos del cultivo de cachama blanca Piaractus orinoquensis, en jaulas lotantes. Rev. U.D.C.A Act. \& Div. Cient. 24(2):e2068. http:// doi.org/10.31910/rudca. v24.n2.2021.2068

Artículo de acceso abierto publicado por Revista U.D.C.A Actualidad \& Divulgación Cientí ica, bajo una Licencia Creative Commons CC BY-NC 4.0

Publicación o icial de la Universidad de Ciencias Aplicadas y Ambientales U.D.C.A, Institución de Educación Superior Acreditada de Alta Calidad por el Ministerio de Educación Nacional.

Recibido: agosto 22 de 2021

Aceptado: noviembre 23 de 2021

Editado por: Helber Adrián Arévalo Maldonado

\section{RESUMEN}

La región de la Orinoquia, se caracteriza por poseer espejos de agua que se mantienen durante todo el año, aportando peces para consumo local. La pesca artesanal constituye la principal actividad económica de sustento de comunidades ribereñas, quienes están siendo amenazadas en su seguridad alimentaria, por la disminución del recurso íctico, debido a múltiples causas atribuidas a la actividad humana. Por lo anterior, sistemas de producción en jaulas flotantes, elaboradas con materiales de bajo costo y ubicadas en cursos de aguas naturales, constituyen una alternativa económica para estas comunidades. El objetivo fue evaluar el rendimiento productivo de la cachama blanca en jaulas flotantes, ubicadas en el río Manacacias, manejando tres densidades: 150,200 y 250 peces $/ \mathrm{m}^{3}$. Se sembraron alevinos en jaulas, con dimensiones de $10,2 \mathrm{~m}^{3}$ ( $\left.3 \times 2 \times 1,7 \mathrm{~m}\right)$. Los peces fueron alimentados con alimento balanceado, durante 126 días. Al final del experimento, los individuos fueron cosechados. Se produjo una biomasa promedio por densidad de $47,42 \mathrm{~kg} / \mathrm{m}^{3}$, en la densidad $150 ; 45,11 \mathrm{~kg} / \mathrm{m}^{3}$, en 200 peces/ $\mathrm{m} 3$ y $79,65 \mathrm{~kg} / \mathrm{m} 3$, en la densidad 250. Su conversión alimenticia fue 1,$1 ; 1,32$ y 0,8 , la sobrevivencia de 83,51, 78,98 y $93,72 \%$, respectivamente. Se concluye, que la cachama blanca puede ser cultivada en jaulas flotantes a altas densidades, obteniendo parámetros productivos óptimos y, adicionalmente, puede ser una fuente de ingresos significativa para las comunidades.

Palabras clave: Densidad de siembra; Especie neotropical; Piscicultura; Río Manacacias; Serrasalmidae. 


\section{ABSTRACT}

The Orinoquia region is characterized by having water bodies that are maintained throughout the year, providing fish for local consumption. Artisanal fishing constitutes the main economic activity for the livelihood of riverine communities, which are being threatened in their food security by the decrease in the fish resource due to multiple causes attributed to human activity. Therefore, production systems in floating cages, made with lowcost materials and located in natural water courses, constitute an economic alternative for these communities. The objective of this work was to evaluate the productive performance of white cachama in floating cages located in the Manacacias river, managing three densities 150, 200 and $250 \mathrm{fish} / \mathrm{m}^{3}$. Fingerlings were sown in cages with dimensions of $10.2 \mathrm{~m}^{3}(3 \times 2 \times 1.7 \mathrm{~m})$. The fish were fed with balanced feed for 126 days. At the end of the experiment the individuals were harvested in three cages for the three densities. An average biomass by density of $47.22 \mathrm{~kg} / \mathrm{m}^{3}$ was produced at density $45.11 \mathrm{~kg} / \mathrm{m}^{3}$ in $200 \mathrm{fish} / \mathrm{m}^{3}$ and $79.65 \mathrm{~kg} / \mathrm{m}^{3}$ at density 250 . Its feed conversion was $1.1,1.32$ and 0.8 , the survival of $83.51,78.98$ and $93.72 \%$ respectively. It is concluded that cachama blanca can be cultivated in floating cages at high densities, obtaining optimal productive parameters and additionally, it can be a significant source of income for the communities.

Keywords: Stocking density; Neotropical fish; Fish farming; Manacacias river; Serrasalmidae.

\section{INTRODUCCIÓN}

En el siglo XXI, se espera aumentar producción para cumplir con las expectativas alimenticias de la creciente población, a través de la producción sostenible, con un mínimo de alteración ambiental (De Silva, 2000). Los sistemas de cultivo en jaulas empleados por los acuicultores son tan diversos como el número de especies que se usan actualmente, que van desde las operaciones familiares y tradicionales (muy común en los países asiáticos), a operaciones de cultivos industriales de salmón y de trucha a gran escala, en el norte de Europa y América (Halwart et al. 2007). En la última década, se observó un crecimiento en la utilización de jaulas en Colombia y de estudios sobre la cría en especies nativas del país.

El orden Characiformes, incluye la mayoría de los peces continentales, que se utilizan para desarrollar planes piscícolas, especialmente, los géneros Colossoma y Piaractus (Granado, 2000). El éxito potencial de una especie nativa, se basa en el análisis de mercado, el rendimiento del crecimiento y de la disponibilidad de los juveniles (Gonçalves De Oliveira et al. 2012). Piaractus orinoquensis (Escobar et al. 2019), conocida como cachama blanca, es la más importante especie nativa en cautiverio, para el desarrollo de pequeñas economías de sustento en Colombia (Pineda S. et al. 2006). Esta especie en sistemas intensivos de producción son altamente dependientes del alimento concentrado, habitualmente, dietas secas, que impactan al medio acuático donde esté ubicado (Molina Domínguez \& Vergara Martín, 2005; Isla Molleda et al. 2019).
Los modelos productivos en jaulas pueden provocar desechos contaminantes por el alimento no consumido, que pasa a través de la jaula y se hunde al fondo del río (Da Silva Cacho et al. 2020). El río Manacacias cuenta con una gran riqueza ictiológica, entre ellas, la cachama, especie endémica de este río, con características digestivas, que permiten un menor impacto ambiental (De Oliveira et al. 2019), al ser trabajadas en jaulas, haciendo uso de las masas de agua existentes (Rowland et al. 2006). Los peces criados en densidades entre 20 y 30 peces $/ \mathrm{m}^{3}$ presentan tasas de crecimiento y de sobrevivencia mayores al $90 \%$, pero un rendimiento que no es afectado por estas condiciones de densidad (Gomes et al. 2000; De Carvalho Gomes et al. 2006).

De esta manera, el objetivo del presente estudio fue evaluar el desempeño productivo de la cachama blanca Piaractus orinoquensis, cultivada en jaulas flotantes.

\section{MATERIALES Y MÉTODOS}

Localización y descripción del área del estudio. Las jaulas, se instalaron en el río Manacacias, Meta, en el punto conocido como ensenada Soplaviento (4¹6'43" N y 7202'47' W). Actualmente, existen comunidades que subsisten con la pesca artesanal en el río; en la época seca, la Autoridad Nacional de Acuicultura y Pesca (AUNAP) realiza vedas para respetar los ciclos reproductivos de los peces, haciendo deficiente la fuente de ingresos de estas poblaciones.

Material biológico. Se utilizaron juveniles de cachama blanca $P$. orinoquensis, con un peso promedio de 4,92 $\pm 1,53 \mathrm{~g}$ y una longitud total de $6,8 \pm 0,55 \mathrm{~cm}$, obtenidos por reproducción artificial y suministrados por piscícolas locales, de la región del departamento del Meta.

Descripción de las unidades de crecimiento (jaulas). El ensayo fue realizado en jaulas flotantes, con dimensiones de $3 \mathrm{~m}$ de largo, 2 $\mathrm{m}$ de ancho y $1,7 \mathrm{~m}$ de profundidad, con un borde libre de $20 \mathrm{~cm}$, con un volumen efectivo de $10 \mathrm{~m}^{3}$. Las jaulas fueron construidas con tubo PVC (Policloruro de vinilo) de 6" pulgadas, malla eslabonada recubierta con PVC de 1" y una estructura metálica con las dimensiones de las jaulas, en forma de cubo, como soporte de la malla de protección a depredadores. Para la flotación de la jaula, se utilizaron envases plásticos cerrados herméticamente de 20 L de capacidad. Para evitar la salida del alimento suministrado, se incorporó una malla de PVC de 8 a $30 \mathrm{~cm}$, de la base superior de la jaula. Todas las jaulas fueron ancladas a una base fija y separadas entre sí, a una distancia de $3 \mathrm{~m}$.

Diseño experimental. Se utilizó un ensayo de tipo experimental, de efecto fijo y aleatorio, para evaluar diferentes densidades de siembra o tratamientos, sobre el crecimiento de cachama blanca. Para tal motivo, se utilizaron densidades de 150 (D1), 200 (D2) y 250 peces $/ \mathrm{m}^{3}$ (D3), con un total de animales de 1.500, 2.000 y 2.500 alevinos por jaula. Cada tratamiento contó con tres repeticiones, constituyendo 9 jaulas o unidades experimentales de crecimiento. Fue suministrado alimento extrusado dos veces al día, 
hasta aparente saciedad, con $34 \%$ de Proteína Cruda (PC), con una duración del ensayo de 180 días (6 meses).

Parámetros de desempeño productivo. Cada 30 días, se realizaron muestreos del $10 \%$ de la población total de la jaula. Para facilitar la manipulación y la disminución del estrés, los animales fueron anestesiados por medio de choque térmico en recipiente con agua y hielo, buscando un efecto de hipotermia. La longitud total (LT) y la longitud estándar (LS), se midieron con un ictiómetro (al milímetro más cercano) y, el peso vivo, con balanza digital $(0,1$ g. Ohaus Scout Pro). Para evaluar el desempeño productivo, se midieron las siguientes variables:

Ganancia en peso $\mathrm{GP}(\mathrm{g})=$ peso final $(\mathrm{g})$ - peso inicial $(\mathrm{g})$.

Ganancia de peso diaria GPD $(\mathrm{g} /$ días = peso final - peso inicial $/$ días del ciclo de producción.

Conversión alimenticia $(\mathrm{CA})=$ consumo de alimento $(\mathrm{g}) /$ ganancia de peso total (g).

Tasa de crecimiento específico en peso TCEP $=[(\operatorname{Ln}$ peso final $/ \mathrm{Ln}$ peso inicial)/ tiempo de cultivo $\mathrm{x} 100]$ ).

Factor de crecimiento relativo $\mathrm{FCR}=$ peso final $(\mathrm{g} /$ longitud total final $(\mathrm{cm})$,

Biomasa final $\left(\mathrm{kg} / \mathrm{m}^{3}\right)$.

Al final del experimento, se determinó la sobrevivencia, utilizando la relación Sobrevivencia $(\%=($ No. final de individuos $/$ No. inicial de individuos) x 100.

Adicionalmente, después del sacrificio fueron determinados los índices corporales: índice viscerosomático (IVS) $=100 *$ (peso de vísceras $(\mathrm{g}) /$ peso corporal $(\mathrm{g})$, índice hepatosomático (IHS) $=100^{*}$ (peso de hígado $(\mathrm{g}) /$ peso corporal $(\mathrm{g})$ ) e índice de grasa visceral $(\mathrm{IGV})=100^{*}$ (peso de grasa visceral $(\mathrm{g}) /$ peso corporal $\left.(\mathrm{g})\right)$.

Calidad de agua. La temperatura del agua y el oxígeno disuelto, se midieron con un sensor (Ecosense ${ }^{\circledR}$ DO200A, YSI, Yellow Springs, USA). El pH, los sólidos totales y la conductividad, se tomaron con el sensor EC500 - ExStik® II impermeable - Extech, Boston USA. El fósforo, alcalinidad, dureza y amonio, se monitorearon una vez al mes, llevando las muestras del laboratorio de calidad de agua, en la Universidad de los Llanos. Las muestras, se tomaron en tres puntos de referencia: $1=$ diez metros antes de la producción, $2=$ en las jaulas, $3=$ diez metros debajo de la producción, teniendo en cuenta la corriente de agua.

Análisis estadístico. Para todos los casos, los valores fueron expresados como media \pm desviación estándar de la media (SD). Se evaluaron los supuestos de homogeneidad y de normalidad de los datos, por medio de las pruebas de Bartlett y Shapiro Wilks. Para evaluar el efecto de cada densidad de siembra sobre los parámetros productivos, se empleó un análisis de varianza
(ANOVA), seguido de una comparación de medias de Tukey. En todos los casos, $\mathrm{p}<0,05$ fue utilizado como criterio estadístico, para revelar diferencias significativas. Los datos fueron analizados con el software Graphpad prism (Versión 8.0).

\section{RESULTADOS Y DISCUSIÓN}

Calidad del agua. Las variables registradas en la investigación no presentaron diferencias significativas antes y después del ensayo. Por lo tanto, los datos se agruparon para dar un estándar de calidad de agua del río Manacacias, que posee características fisicoquímicas y limnológicas semejantes a los ríos de altillanura de la cuenca del Orinoco. El oxígeno disuelto durante el experimento en las tres zonas de muestreo osciló entre 4,53 y 4,74 mg/L y la temperatura entre 26,70 a $30,03{ }^{\circ} \mathrm{C}$ (Figura 1. Variables, como el $\mathrm{pH}$, la alcalinidad, la conductividad, los nitratos y el amonio, se mantuvieron en rangos similares, sin presentar diferencias estadistas significativas. Por su parte, los sólidos totales y fosfato fósforo mostraron una pequeña variación de valores en la zona tres, posiblemente, asociada a mayor presencia de materia orgánica en el río (Tabla 1).

El lugar escogido para este estudio fue en un meandro del río Manacacias, que mostró un $\mathrm{pH}$ ligeramente ácido, bajos valores de nutrientes y de conductividad y una alta transparencia (Yossa, 2018). El oxígeno disuelto mostró altas concentraciones en comparación con otras fuentes de agua del país (Carrera \& Valbuena-Villareal, 2015), lo cual, brinda una ventaja para establecer altas densidades de peces en jaulas. Al cotejar el resultado de este estudio con uno realizado en Venezuela con la misma especie (Granado, 2000), se evidenció las bondades del oxígeno, con una sobrevivencia mayor al $92 \%$ y un rendimiento de $17,7 \mathrm{Kg} / \mathrm{m}^{3}$.

Crecimiento y rendimiento. Los resultados mostraron que la densidad D3 para la GP fue mayor, con un valor promedio de $341,09 \pm 1,33 \mathrm{~g}$, con diferencias significativas respecto a las densidades $\mathrm{D} 1$ y D2. Igualmente, se observaron resultados positivos en los parámetros zootécnicos, a pesar de una diferencia marcada entre las densidades manejadas en D1, D2 y D3, posiblemente, relacionada con los lugares donde se ubicaron las tres jaulas. La D3 recibió las corrientes directas del río, con mayor cantidad de material "verde", que ingresaba a la jaula, mejorando el ciclaje de nutrientes (Mulholland, 1996).

En todos los tratamientos, el peso promedio fue mayor a $250 \mathrm{~g}$, valor inferior a lo descrito por Poleo et al. (2011), cultivando cachama blanca, a densidades de siembra de 31,25 peces $/ \mathrm{m}^{3}$; sin embargo, estos resultados son mayores a lo observado en el crecimiento de otras especies, como yaque Leiarius marmoratus, a una densidad de 30 peces $/ \mathrm{m}^{3}$ (Murillo-Pacheco et al. 2012) y de 60 peces $/ \mathrm{m}^{3}$, en un bi-cultivo de cachama blanca y tilapia nilótica (Brú-Cordero et al. 2017).

El aumento en peso por unidad de tiempo o tasa específica de crecimiento fue similar para las diferentes densidades evaluadas (Tabla 2). Reátegui Acosta et al. (2017) reportan valores cercanos 


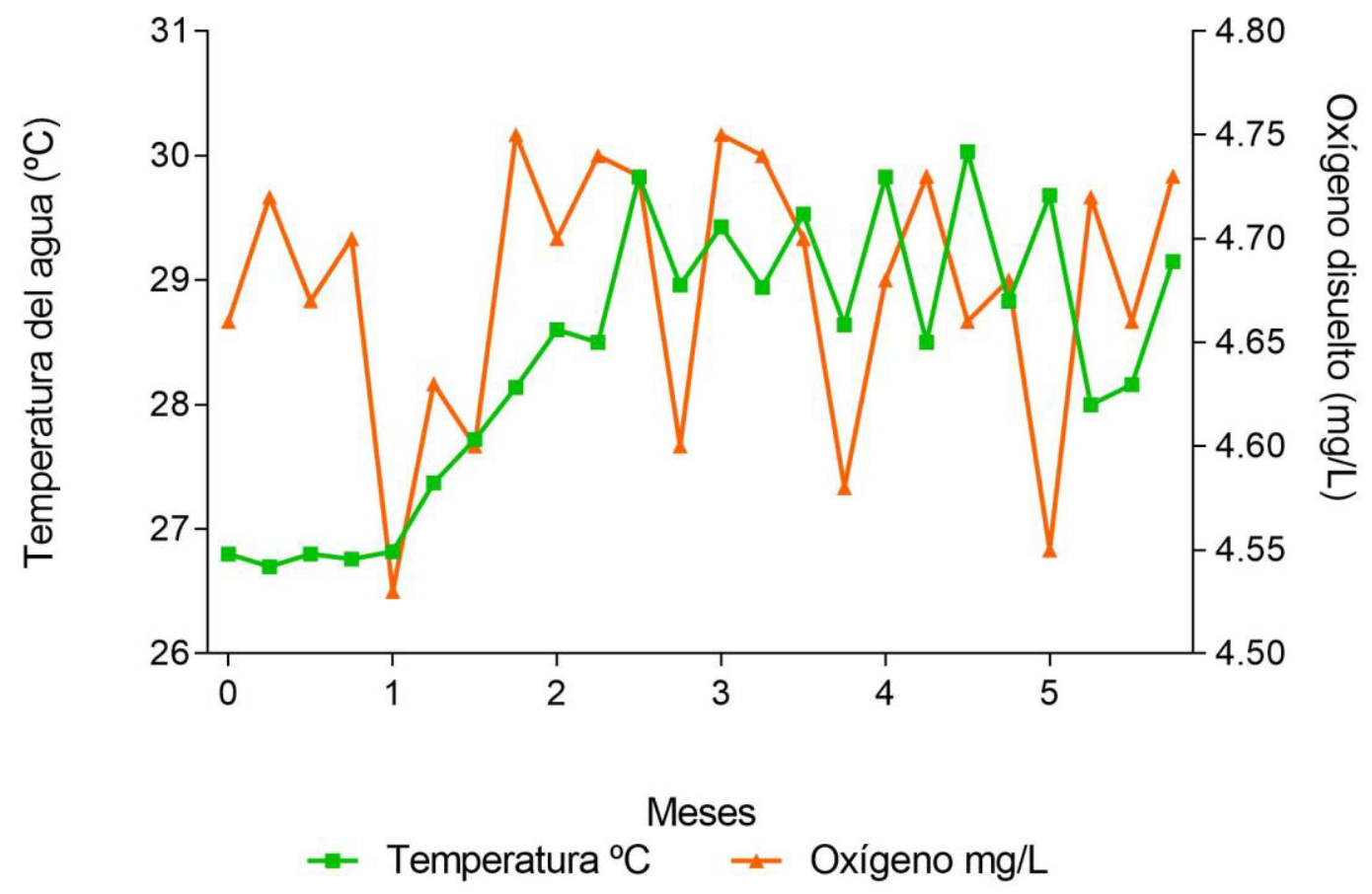

Figura 1. Comportamiento del oxígeno disuelto y la temperatura del agua, en el río Manacacias, en la zona de cultivo de Piaractus orinoquensis, cultivado durante 180 días, en un sistema de jaulas flotantes, a diferentes densidades. Valores mostrados como media.

Tabla 1. Variables ambientales del río Manacacias, en la zona de cultivo de Piaractus orinoquensis, cultivado durante 180 días, en un sistema de jaulas flotantes, a diferentes densidades de siembra. orinoquensis, cultivado durante 180 días, en un sistema de jaulas flotantes, a diferentes densidades. Valores mostrados como media.

\begin{tabular}{|c|c|c|c|}
\hline Variable & Zona 1 & Zona 2 & Zona 3 \\
\hline $\mathbf{p H}$ & $6,53 \pm 0,26 \mathrm{a}$ & $6,43 \pm 0,31 \mathrm{a}$ & $6,41 \pm 0,25 \mathrm{a}$ \\
\hline $\begin{array}{c}\text { Sólidos totales } \\
(\mathbf{m g} / \mathbf{L})\end{array}$ & $3,95 \pm 0,99 \mathrm{bc}$ & $5,61 \pm 1,90 \mathrm{a}$ & $4,80 \pm 1,47 \mathrm{ab}$ \\
\hline $\begin{array}{c}\text { Amonio } \\
(\mathbf{m g} / \mathbf{L})\end{array}$ & $0,17 \pm 0,10 \mathrm{a}$ & $0,16 \pm 0,08 \mathrm{a}$ & $0,16 \pm 0,09 \mathrm{a}$ \\
\hline $\begin{array}{c}\text { Dureza } \\
(\mathbf{m g} / \mathbf{L})\end{array}$ & $3,12 \pm 0,32 \mathrm{a}$ & $3,26 \pm 0,37 \mathrm{a}$ & $3,16 \pm 0,28 \mathrm{a}$ \\
\hline $\begin{array}{c}\text { Conductividad } \\
(\boldsymbol{\mu s} / \mathbf{c m})\end{array}$ & $7,94 \pm 0,98 \mathrm{a}$ & $7,56 \pm 1,4 \mathrm{a}$ & $8,35 \pm 1,59 \mathrm{a}$ \\
\hline $\begin{array}{c}\text { Alcalinidad } \\
(\mathbf{m g} / \mathbf{L} \mathbf{C a C O})\end{array}$ & $4,43 \pm 0,86 \mathrm{a}$ & $4,56 \pm 0,77 \mathrm{a}$ & $4,45 \pm 0,70 \mathrm{a}$ \\
\hline $\begin{array}{c}\text { Nitratos } \\
(\mathbf{m g} / \mathbf{L} \mathbf{N})\end{array}$ & $0,08 \pm 0,05 \mathrm{a}$ & $0,08 \pm 0,06 \mathrm{a}$ & $0,08 \pm 0,05 \mathrm{a}$ \\
\hline $\begin{array}{c}\text { Fosfatos } \\
(\mathbf{m g} / \mathbf{L})\end{array}$ & $0,33 \pm 0,09 \mathrm{~b}$ & $0,25 \pm 0,12 \mathrm{~b}$ & $0,40 \pm 0,18 \mathrm{a}$ \\
\hline
\end{tabular}

Valores mostrados como: media \pm desviación estándar.

Las diferentes letras en las variables entre columnas indican diferencias significativas $(\mathrm{p}<0,05)$.

para la especie 1,622 $\pm 0,292 \%$, a una densidad de 60 peces/ $\mathrm{m}^{3}$, cultivada en jaulas flotantes de $1,5 \mathrm{~m} 3$; sin embargo, estos valores estimados son menores que 0,52 \pm 0,001 \%, informado por Mercado Burgos et al. (2006), para el cultivo en jaulas flotantes de Brycon sinuensis, a una densidad de 25 peces $/ \mathrm{m}^{3}$ y $0,8 \pm 0,002$, reportado para Pimelodus grosskopfii, a una densidad de 25 peces/ 
$\mathrm{m}^{3}$ y alimentado con $25 \%$ de PB (Carrera \& Valbuena-Villareal, 2015). Lo anterior permite inferir que el crecimiento en peso está condicionado a diferentes variables, como la densidad de siembra, afectando, de forma irregular o positiva, el desempeño productivo de la especie.
La densidad D3 produjo la mayor biomasa (Tabla 2). Respecto a los requerimientos de proteína en cachama blanca, se reportan hasta $31 \%$ PC, con digestibilidades variables, según la fuente (VásquezTorres et al. 2012). La cachama tiene hábitos de alimentación omnívora, con tendencia al consumo de frutas, semillas y hojas

Tabla 2. Índices de producción de Piaractus orinoquensis, cultivado durante 180 días, en un sistema de jaulas flotantes, a diferentes densidades de siembra. orinoquensis, cultivado durante 180 días, en un sistema de jaulas flotantes, a diferentes densidades. Valores mostrados como media.

\begin{tabular}{|c|c|c|c|}
\hline \multirow{2}{*}{ Índices de producción } & \multicolumn{3}{|c|}{ Densidad (peces $\left./ \mathrm{m}^{3}\right)$} \\
\hline & D1-150 & $\mathrm{D} 2-200$ & D3- 250 \\
\hline Peso inicial (g) & \multicolumn{3}{|c|}{$4,92 \pm 1,53$} \\
\hline Longitud total inicial $(\mathrm{cm})$ & \multicolumn{3}{|c|}{$6,8 \pm 0,55$} \\
\hline GP (g) & $271,62 \pm 2,43 c$ & $290,63 \pm 1,06 \mathrm{~b}$ & $341,09 \pm 1,33 \mathrm{a}$ \\
\hline GPD (g) & $1,51 \pm 0,01 \mathrm{a}$ & $1,61 \pm 0,00 \mathrm{a}$ & $1,87 \pm 0,00 \mathrm{a}$ \\
\hline CA & $1,1 \pm 0,0 \mathrm{a}$ & $1,2 \pm 0,0 \mathrm{a}$ & $0,9 \pm 0,0 \mathrm{a}$ \\
\hline TECP (\%) & $0,157 \pm 0,0 \mathrm{a}$ & $0,155 \pm 0,0 \mathrm{a}$ & $0,151 \pm 0,0 \mathrm{a}$ \\
\hline FCR & $40,55 \pm 0,35 \mathrm{~b}$ & $43,33 \pm 0,15 \mathrm{ab}$ & $50,72 \pm 0,19 a$ \\
\hline Biomasa final $\left(\mathrm{kg} / \mathrm{m}^{3}\right)$ & $47,22 \pm 8,73 \mathrm{~b}$ & $45,11 \pm 3,33 \mathrm{~b}$ & $79,65 \pm 0,98 \mathrm{a}$ \\
\hline Sobrevivencia (\%) & $83,51 \pm 0,19 b$ & $78,98 \pm 3,34 \mathrm{~b}$ & $93,72 \pm 1,63 a$ \\
\hline
\end{tabular}

GP (Ganancia de peso), GPD (Ganancia Diaria de peso), CA (Conversión alimenticia), TECP (Tasa específica de crecimiento en peso), FCR (Factor de crecimiento relativo).

Valores mostrados como media \pm desviación estándar.

Las diferentes letras en las variables entre columnas indican diferencias significativas $(p<0,05)$

(Araujo-Lima \& Goulding, 1997. La biomasa final en promedio fue de $572,3 \pm 196,39 \mathrm{~kg} / \mathrm{jaula}$, resultado oportuno, que muestra la adaptación de la cachama al cultivo en jaula (Rodrigues Silva et al. 2007). Comparando este estudio con lo reportado para Brycon sp. (Ramos Tortolero et al. 2010), usando densidades de 100, 150, 200 y 250 peces $/ \mathrm{m}^{3}$, los resultados en las biomasas finales fueron similares.

Como se observa en la tabla 2, la CA presentó valores cercanos entre sí para las diferentes densidades; por su parte, la sobrevivencia fue mayor en la densidad D3, mostrando diferencias significativas. La CA obtenida en este trabajo fue menor, en comparación con otros trabajos similares con tambaqui (Colossoma macropomum), donde obtuvieron un valor de 1,8 (Araujo-Lima \& Goulding, 1997); cachama blanca, con 3,0 y 1,2, respectivamente (BrúCordero et al. 2017; Granado, 2000) y en pirarucu (Arapaima gigas), de 1,2 (Gonçalves De Oliveira et al. 2012). La CA tuvo una relación directa con la densidad, siendo D3 y D1 más eficientes, para el aprovechamiento del alimento suministrado. La justificación de este resultado, se puede deber a que los individuos de la D2 tuvieron mayor material externo dentro de la jaula, como hojas, semillas y peces de menor tamaño.

En cuanto a los índices corporales, se observaron diferencias significativas entre las densidades (Figura 2 a-c. La grasa visceral de mayor valor fue observada en la densidad D3, mostrando diferencias significativas con respecto a las otras densidades. Por su parte, el índice Hepatosomático muestra que la D2 fue mayor frente a los otros tratamientos y, estadísticamente, diferentes. El mayor porcentaje de índice viscerosomático fue observado para la
D1, presentando diferencias significativas respecto a las D2 y D3.

El factor de condición expresa el grado de bienestar de un organismo con relación al medio en que vive y está relacionado con la tasa de crecimiento y el nivel de confort de los animales (De Carvalho Gomes et al. 2006; Poleo et al. 2011; Carrera \& Valbuena-Villareal, 2015). Los índices corporales, Viscerosomático (IVS), Hepatosomático (IHS) y de Grasa Visceral (IGV) están vinculados directamente con el factor de condición e inversamente con el grado de madurez del individuo (Ramirez-Merlano et al. 2011; Cardia \& Lovatelli, 2015). Los bajos valores de los índices hepatosomático y viscerosomático están condicionados por las características anatómicas de la especie, mientras que en capaz (Pimelodus grosskopfii) (Carrera \& Valbuena-Villareal, 2015) y en yaque (Poleo et al. 2011), dichos valores son mayores a lo reportado en este trabajo.

La estrategia de éxito en este tipo de producción es el manejo adecuado de la densidad de siembra (Cardia \& Lovatelli, 2015), de acuerdo con las condiciones del entorno, para el mejoramiento de la conversión alimenticia.

En conclusión, los resultados de este estudio, indican el uso factible de altas densidades en el cultivo de cachama blanca, en sistemas de jaulas flotantes, usando una densidad de siembra de 250 peces/ $\mathrm{m} 3$, siendo una alternativa prometedora en la producción de esta especie, en jaulas flotantes, instaladas en ríos del departamento del Meta (Colombia). Por otro lado, este sistema es un modelo productivo viable para las comunidades ribereñas, permitiendo su implementación, a bajos costos de inversión. 

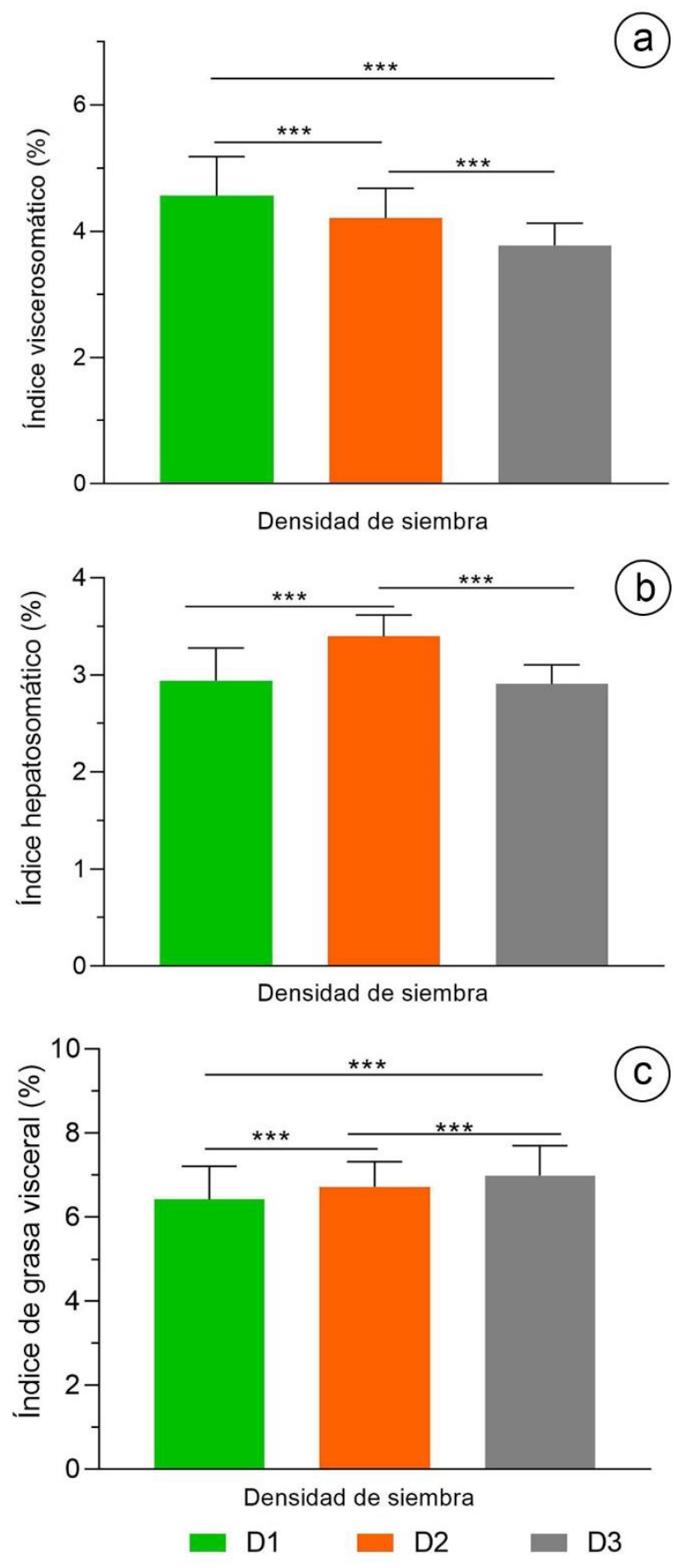

Figura 2. Índices corporales de Piaractus orinoquensis, cultivado durante 180 días, en un sistema de jaulas flotantes, a diferentes densidades de siembra. a. IVS=Índice viscerosomático; b. IHS=Índice hepatosomático; c. IGV= Índice de grasa visceral.

Valores mostrados como media \pm Desviación estándar.

***variables diferenciadas y compartidas en las columnas indican diferencias significativas estadísticamente $(\mathrm{p}<0.05)$.

Agradecimientos. Este estudio fue apoyado por la asociación ASOPESGA del municipio de Puerto Gaitán y la Corporación KOTSALA. Conflictos de intereses: El manuscrito fue preparado y revisado con la participación de todos los autores, quienes declaramos que no existe ningún conflicto de intereses que ponga en riesgo la validez de los resultados presentados.

\section{REFERENCIAS}

1. ARAUJO-LIMA, C.; GOULDING, M. 1997. So fruitful a fish: Ecology, conservation, and aquaculture of the Amazon's tambaqui. Columbia University Press. 191p. 
2. BRÚ-CORDERO, S.B.; PERTÚZ-BUELVAS, V.M.; AYAZOGENES, J.E.; ATENCIO-GARCÍA, VJ.; PARDOCARRASCO, S.C. 2017. Bicultivo en biofloc de cachama blanca Piaractus brachypomus y tilapia nilótica Oreochromis niloticus alimentadas con dietas de origen vegetal. Rev. Med. Vet. Zoot. 64(1):44-60.

https://doi.org/10.15446/rfmvz.v64n1.65824

3. CARDIA, F.; LOVATELLI, A. 2015. Aquaculture operations in floating HDPE cages. A field handbook. FAO Fisheries and Aquaculture Technical Paper 593. Food and Agriculture Organization of the United Nations And Ministry of Agriculture of the Kingdom of Saudi Arabia, Rome. 149p.

4. CARRERA, S.; VALBUENA-VILLAREAL, R.D. 2015. Desempeño productivo del capaz (Pimelodus grosskopfii, Steindachner, 1879) bajo diferentes densidades de siembra y de contenido de proteína en la dieta. Intropica: Revista del Instituto de Investigaciones Tropicales (Colombia). 10:20-27.

5. DA SILVA CACHO, J.C.; TEIXEIRA DE MOURA, R.S.; HENRY-SILVA, G.G. 2020. Influence of Nile tilapia (Oreochromis niloticus) fish farming in net cages on the nutrient and particulate matter sedimentation rates in Umari reservoir, Brazilian semi-arid. Aquaculture Reports (Netherlands). 17:100358.

http://doi.org/10.1016/j.aqrep.2020.100358

6. DE CARVALHO GOMES, L.; CAMPOS CHAGAS, E.; MARTINS-JUNIOR, H.; ROUBACH, R.; AKIFUMI ONO, E.; DE PAULA LOURENÇO, J.N. 2006. Cage culture of tambaqui (Colossoma macropomum) in a central Amazon floodplain lake. Aquaculture (Netherlands). 253(1-4):374-384.

http://doi.org/10.1016/j.aquaculture.2005.08.020

7. DE OLIVEIRA, M.I.B.; DE MATOS, L.V.; DA SILVA, L.A.; CHAGAS, E.C.; DA SILVA, G.S.; GOMES, A.L. 2019. The digestive tube of Piaractus brachypomus: gross morphology, histology/histochemistry of the mucosal layer and the effects of parasitism by Neoechinorbynchus sp. J. Fish. Biol. (Great Britain). 94(4):648-659.

http://doi.org/10.1111/jfb.13934

8. DE SILVA, S.S. 2000. A global perspective of aquaculture in the new millennium. In: Subasinghe, R.P.; Bueno, P.B.; Phillips, M.J.; Hough, C.; McGladdery, S.E.; Arthur, J.R. (eds.). Technical proceedings of the conference on aquaculture in the third millennium. Network of Aquaculture Centres in Asia-Pacific. Bangkok, Thailand. p.431-459.

9. ESCOBAR, M.D.; OTA, R.P.; MACHADO-ALLISON, A.; ANDRADE-LÓPEZ, J.; FARIAS I.P.; HRBEK, T. 2019. A new species of Piaractus (Characiformes: Serrasalmidae) from the Orinoco Basin with a redescription of Piaractus brachypomus. J. Fish. Biol. 95(2):411-427.

http://doi.org/10.1111/jfb.13990

10. GOMES, L.; BALDISSEROTTO, B.; SENHORINI, J.A. 2000. Effect of stocking density on water quality, survival, and growth of larvae of the matrinxa, Brycon cephalus (Characidae), in ponds. Aquaculture (Netherlands). 183(1-2):73-81. http://doi.org/10.1016/S0044-8486(99)00288-4

11. GONÇALVES DE OLIVEIRA, E.; BANHOS PINHEIRO, A.; QUEIROZ DE OLIVEIRA, V.; MELO DA SILVA JÚNIOR, A.R.; GAZZINEO DE MORAES, M.G.; CASTELO BRANCO ROCHA, I.R.; ROCHA DE SOUSA, R.; FARIAS COSTA, F.H. 2012. Effects of stocking density on the performance of juvenile pirarucu (Arapaima gigas) in cages. Aquaculture (Netherlands). 370-371:96-101. http://doi.org/10.1016/j.aquaculture.2012.09.027

12. GRANADO, A. 2000. Efecto de la densidad de cultivo sobre el crecimiento del morocoto, Piaractus brachypomus, Cuvier, 1818 (Pisces: Characiformes). Saber (Venezuela). 12(2):3-7.

13. HALWART, M.; SOTO, D.; ARTHUR, J.R. 2007. Cage aquaculture: regional reviews and global overview. FAO Fisheries and Aquaculture Technical Paper 498. Food and Agriculture Organization of the United Nations and Ministry of Agriculture of the Kingdom of Saudi Arabia, Rome. 241p.

14. ISLA MOLLEDA, M.; FLORES GUTIÉRREZ, E.R.; TORE LUNESTAD, B.; KARLSEN, O; RODRÍGUEZ CRUZATA, P.; VEGA, A.B.; COELLO, D.M. 2019. Estado ambiental de la zona donde se desarrolló el cultivo de cobia (Rachycentron canadum) en jaulas flotantes, bahía de cochinos, Cuba. Rev. cubana de Investigaciones Pesqueras (Cuba). 36(2):73-81.

15. MERCADO BURGOS, I.; GARCÍA, J.L.; ROSADO CÁRCAMO, R.; OLAYA-NIETO, C.W.; SEGURA GUEVARA, F.F.; BRÚ-CORDERO, S.; TORDECILLAPETRO, G. 2006. Cultivo de dorada (Brycon sinuensis Dahl, 1955) en jaulas flotantes a diferentes niveles de proteína. Rev Colombiana de Ciencias Pecuarias (Colombia). 19(2):204-211.

16. MOLINA DOMÍNGUEZ, L.; VERGARA MARTÍN, J.M. 2005. Impacto ambiental de jaulas flotantes: estado actual de conocimientos y conclusiones prácticas. Boletín. Instituto Español de Oceanografía (España). 21(1-4):75-81.

17. MULHOLLAND, P.J. 1996. Role in Nutrient Cycling in Streams. In: Stevenson, R.J. (ed.). Algal ecology: freshwater benthic ecosystems. Elsevier Inc. p.609-639.

18. MURILLO-PACHECO, R.; CRUZ-CASALLAS, N.E.; RAMIREZ-MERLANO, J.; MARCIALES-CARO, L.; MEDINA-ROBLES, V.M.; CRUZ-CASALLAS, P. 2012. 
Efecto del nivel de proteína sobre el crecimiento del yaque Leiarius marmoratus (Gill, 1870) bajo condiciones de cultivo. Rev. Orinoquia (Colombia). 16:52-61.

https://doi.org/10.22579/20112629.146

19. PINEDA S., H.; OLIVERA A., M.; URCUQUI I., S.; TRUJILLO B., E.; BUILES G., J.J. 2006. Evaluación del polimorfismo por microsatélites en individuos de Piaractus brachypomus (Characidae, Serrasalminae) provenientes del río Meta, Colombia. Rev. Colom. Cienc. Pecua. (Colombia). 19:66-69.

20. POLEO,G.;ARANBARRIO,J.V.;MENDOZA,L.;ROMERO, O. 2011. Cultivo de cachama blanca en altas densidades y en dos sistemas cerrados. Pesquisa Agropecuária Brasileira (Brasil). 46(4):429-437.

http://doi.org/10.1590/S0100-204X2011000400013

21. RAMIREZ-MERLANO, J.; MEDINA-ROBLES, V.; CRUZ-CASALLAS, P. 2011. Variación estacional de las características seminales del bagre rayado Psendoplatystoma metaense (Telostei, pimelodidae). Rev. MVZ Córdoba (Colombia). 16(1):2336-2348.

https://doi.org/10.21897/rmvz.292

22. RAMOS TORTOLERO, S.A.; FIGUEREDO SOARES, M.C.; SUAREZ MERA, P.A.; FARIA MONTEIRO, J.M. 2010. Efeito da densidade de estocagem no crescimento do matrinxã, Brycon amaz̧onicus (Spix \& Agassiz, 1829) em gaiolas de pequeno volume. Rev. Brasileira de Engenharia de Pesca. 5(1):81-92. https://doi.org/10.18817/repesca.v5i1.294
23. REÁtEGUI ACOSTA, C.R.; OLIVA PAREDES, R.; VILLEGAS PANDURO, P.P.; VARGAS FLORES, J.I. 2017. Efecto de la densidad de siembra en el desempeño productivo y parámetros hematológicos de juveniles de Piaractus brachypomus "paco" cultivados en jaulas flotantes en la laguna Yarinacocha. Rev de Investigación Científica - Pucallpa. (Perú). 2(2)

https://doi.org/10.37292/riccva.v2i02.58

24. RODRIGUES SILVA, C.; CARVALHO GOMES, L.; RODRIGUES BRANDÃO, F. 2007. Effect of feeding rate and frequency on tambaqui (Colossoma macropomum) growth, production and feeding costs during the first growth phase in cages. Aquaculture (Netherlands). 264(1-4):135-139.

https://doi.org/10.1016/j.aquaculture.2006.12.007

25. ROWLAND, S.J.; MIFSUD, C.; NIXON, M.; BOYD, P. 2006. Effects of stocking density on the performance of the Australian freshwater silver perch (Bidyanus bidyanus) in cages. Aquaculture (Netherlands). 253(1-4):301-308. http://doi.org/10.1016/j.aquaculture.2005.04.049

26. VÁSQUEZ-TORRES, W.; HERNÁNDEZ-ARÉVALO, G.; GUTIÉRREZ-ESPINOSA, M.C.; YOSSA, M.I. 2012. Effects of dietary protein level on growth and serum parameters in cachama (Piaractus brachypomus). Rev. Colom. Cienc. Pecua. (Colombia). 25:450-461.

27. YOSSA, M. 2018. Parámetros limnológicos del área de influencia del cultivo de cachama (Piaractus brachypomus) en jaulas. Rev. Orinoquia. (Colombia). 22(1):41-47.

http://doi.org/10.22579/20112629.47 